reached areas not targeted for media coverage, the pilot campaign ran after Christmas when fluctuations in screening activity are considerable and age-extension was underway in some areas.

Conclusion At the end of January 2012, the Government launched a 9 -week national bowel cancer awareness campaign. Providers have been urged to plan for a $50 \%$ increase in GP referrals during the campaign and for a sustained increase in colonoscopy demand over the next 5 years. This analysis of local screening activity during the pilot campaign, however, suggests that the direct effect of the national NAEDI campaign on bowel cancer screening hub activity is likely to be modest.

Competing interests None declared.

\section{PWE-093 THE NHS BOWEl CANCER SCREENING PROGRAMME, SOUTHERN HUB-SCREENING ACTIVITY AND OUTCOMES}

doi:10.1136/gutjnl-2012-302514d.93

H Seaman, M Young, ${ }^{*}$ S Halloran. NHS Bowel Cancer Screening Programme, Southern Hub, Guildford, UK

Introduction As part of the NHS Bowel Cancer Screening Programme (BCSP) in England, every man and woman registered with a GP, living in England and aged 60-74 years, is invited to take part in screening every 2 years. The BCSP Southern Hub, hosted by the Royal Surrey County Hospital and one of five Hubs in England, serves a total population of about 14.4 million people and manages the screening activity in the south of England (excluding London). The Southern Hub handles nearly one million gFOB test kits every year. Here we provide a high-level overview of screening activity and outcomes for the Southern Hub since the Programme's launch in 2006.

Methods Screening invitees are sent a guaiac-based faecal occult blood (gFOB) test kit and asked to provide a faecal sample. Test kits are returned to the Hub for analysis. Participants with a positive ("abnormal") test are referred to a Specialist Screening Practitioner (SSP) for further assessment and investigation (usually colonoscopy) at one of 17 Screening Centres. All screening activity, including invitation uptake, gFOB test results, SSP referrals and colonoscopy outcomes are stored on a dedicated database-the Bowel Cancer Screening System (BCSS). The BCSS provides a rich source of data for observational analysis.

Results The uptake of screening invitations (the proportion of invitees that was adequately screened) is approximately $56 \%$ overall. Uptake is generally higher for women ( $61 \%$ vs $55 \%$ ), although improves with age in men. The proportion of positive test kits ("positivity") is higher for men (2.6\%) than for women (1.6\%) at all ages. The number of colonoscopies performed at the Screening Centres has increased over time. About $40 \%$ of the screened population that tests positive and undergoes colonoscopy has significant neoplasia (cancer, high- or intermediate-risk adenomas). The prevalence of significant neoplasia is greater in men and increases with age. The proportion of significant neoplasia detected in screening episode 2 is lower than in episode 1, reflecting successful detection of lesions in the first episode.

Conclusion The BCSS data are encouraging and indicate that the BCSP in England is likely to achieve its goal of reducing mortality from bowel cancer.

Competing interests None declared.

\section{PWE-094 UNDERSTANDING NON-PARTICIPATION IN BOWEL CANCER SCREENING: A QUALITATIVE STUDY}

doi:10.1136/gutjnl-2012-302514d.94

${ }^{1} \mathrm{~N}$ J Hall, ${ }^{*} \mathrm{G}$ P Rubin, ${ }^{2} \mathrm{D}$ Weller, ${ }^{3} \mathrm{~J}$ Wardle, ${ }^{1} \mathrm{C}$ Dobson, ${ }^{4} \mathrm{M}$ Ritchie, ${ }^{1,5,6} \mathrm{C}$ Rees.

${ }^{1}$ School of Medicine and Health, Durham University, Stockton, UK; ${ }^{2}$ Community Health
Sciences, University of Edinburgh, Edinburgh, UK; ${ }^{3}$ Health Behaviour Unit, University College London, London, UK; ${ }^{4}$ South of Tyne Bowel Cancer Screening Centre, Queen Elizabeth General Hospital, Gateshead, UK; ${ }^{5}$ Gastroenterology, South Tyneside NHS Foundation Trust, South Shields, UK; ${ }^{6}$ Northern Region Endoscopy Group, UK

Introduction Uptake of the national bowel cancer screening programme (BCSP), at $52 \%$, needs to be improved or at least maintained if the screening programme is to achieve projected reductions in mortality and morbidity. Understanding the origins of non-participation is therefore important. This study used qualitative methods to explore the beliefs and experiences of individuals who had not responded either to their screening invitation or reminder.

Methods In-depth qualitative interviews with volunteers were used to enable maximum opportunity for exploration and inductive hypothesis generation. Non-participation was defined as having refused all of the invitations and reminders for FOB test screening received from the North East Hub of the BCSP at the time of contact. Interviewees were purposefully sampled to allow for diversity in terms of gender, geographical location and socioeconomic status. Data collection and analysis were carried out using strategies consistent with the principles of grounded theory with an emphasis on the constant comparison method. Data collection and analysis took place concurrently and continued until saturation (27 interviews)

Results The interviews provided an in-depth understanding of a range of reasons and circumstances surrounding non-participation, including contextual and environmental influences as well as factors specific to the screening test. The nature of the data also allowed an appreciation of the potential for changes in beliefs, awareness and intention over time. Most of the interviewees had positive attitudes towards the BCSP, even those who did not feel screening was appropriate for them or who did not wish to take part. Many had intended to take part or intended to take part in the future. The main emergent categories included: practicalities of screening, value of screening, knowledge and awareness, risk perceptions, intention, embarrassment, good "citizenship", guilt, control, and the influence of others.

Conclusion A range of different approaches may be required to improve uptake, depending on the experiences, circumstances, beliefs and existing levels of intention of non-participants. Many of the interviewees in this study reported an intention to take part in future screening rounds. This group might be responsive to repeat invitations, reminders, and aids to making the test practical. Individuals who are opposed to screening (or BCS in particular) may have been less willing to be interviewed. Research is needed to ascertain whether different groups of non-responders require different approaches to intervention.

Competing interests None declared.

\section{PWE-095 ROLE OF RESTRICTED FLUID THERAPY IN PATIENTS UNDERGOING LAPAROSCOPIC AND OPEN COLORECTAL SURGERY: A SYSTEMATIC REVIEW AND META-ANALYSIS OF PUBLISHED RANDOMISED CONTROLLED TRIALS}

doi:10.1136/gutjnl-2012-302514d.95

M Sajid, N Ladwa,* P Sains, M K Baig. Department of General and Laparoscopic Colorectal Surgery, Worthing Hospital, Worthing, UK

Introduction Sub-optimal fluid therapy during peri-operative time period may influence the postoperative mortality and morbidity. The aim of this article is to systematically review the randomised trials analysing the restricted fluid therapy (RFT) and non-restricted 
fluid therapy (NRFT) in patients undergoing laparoscopic and open colorectal surgery.

Methods A simple model was applied to evaluate the various variables reported in the published randomised, controlled trials comparing the role of RFT and NRFT by the use of principles of meta-analysis. The primary outcome measure was postoperative morbidity. Secondary endpoints were mortality and hospital stay. A random effects model was applied.

Results Seventeen randomised, controlled trials on 2165 patients were included. The incidence of postoperative morbidity (OR 0.84; $95 \% \mathrm{CI} 0.57$ to $1.24 ; \mathrm{z}=0.90 ;=0.37$ ) and mortality (OR $0.93 ; 95 \% \mathrm{CI}$ 0.47 to $1.84 ; z=0.20 ;=0.84$ ) was statistically similar following the use of either RFT or NRFT. In addition, both techniques of fluid therapy were associated with similar length of hospital stay (standardised mean difference, $-0.12 ; 95 \%$ CI -0.55 to 0.31 ; $\mathrm{z}=0.53 ; \mathrm{p}=0.59$ ).

Conclusion This meta-analysis suggests that RFT in patients undergoing laparoscopic and open colorectal surgery does not offer any advantage over NRFT.

Competing interests None declared.

\section{PWE-096 THE INVESTIGATION AND MANAGEMENT OF COLOVESICAL FISTULAE IN THE MODERN ERA-A SINGLE INSTITUTIONS 12-YEAR EXPERIENCE}

doi:10.1136/gutjnl-2012-302514d.96

N Ladwa, ${ }^{*}$ M Sajid, M McFall, A Miles, P Sains, M K Baig. Department of General and Laparoscopic Colorectal Surgery, Worthing Hospital, Worthing, UK

Introduction This aim of this study is to review how investigation and management of colovesical fistulae has progressed in our institution over a 12-year period and to propose a clear protocol to ensure prompt diagnosis and treatment in the future.

Methods A retrospective case note review was conducted of all patients with colovesical fistulae who underwent definitive surgery over a 12-year period. Variables collected include patient demographics, symptoms, investigations, operative data, histology, complications and length of stay.

Results 56 patients (38 male) underwent operative intervention for colovesical fistula. The most common symptoms are pneumaturia $(69 \%)$, faecaluria $(32 \%)$ and symptoms associated with recurrent UTIs $(68 \%)$. Cystoscopy was the most accurate test to identify fistulae (91\%) followed by CT (60\%) and barium enema (31\%). Two patients were unfit for major surgery and underwent palliative loop colostomies. The most common pathology was diverticular disease. Of the 54 remaining patients, $45 \%$ underwent laparoscopic resection with a conversion rate of $33 \%$ (due to adhesions or multiple abscesses). Sigmoid colectomy, (52\%) anterior resection (30\%) and hartmanns $(9 \%)$ are the most common procedures performed. Bladder repair was required in $25 \%$ of cases with a further $16 \%$ requiring partial resection. All patients received a postoperative cystogram to ensure bladder had healed and $70 \%$ of patients were defunctioned to protect the anastomosis. There was no mortality reported peri-operatively; the anastomotic leak rate was $5 \%$ and recurrence rate was $5 \%$. Median postoperative stay was 12.5 days (range 4-91) in the laparoscopic group and 16 days (range 6-62) in the open group.

Conclusion Surgical management for colovesical fistulae is effective and safe. Laparoscopic resections are increasing in popularity and deliver encouraging results comparable to open resection. A large multi-centre randomised controlled trial is required to validate its potential benefits over open surgery.

Competing interests None declared.

\section{PWE-097 CLASSIFICATION OF PSEUDOMYXOMA PERITONEI AS LOW OR HIGH GRADE ACCORDING TO THE WHO CRITERIA IS PROGNOSTICALLY SIGNIFICANT}

doi:10.1136/gutjnl-2012-302514d.97

${ }^{1} \mathrm{~N}$ Carr, ${ }^{*}{ }^{2} \mathrm{~B}$ Moran, ${ }^{2} \mathrm{~T}$ Cecil, ${ }^{2} \mathrm{~K}$ Chandrakumaran, ${ }^{3} \mathrm{C}$ llesley, ${ }^{4} \mathrm{~A}$ Mirnezami, ${ }^{2} \mathrm{~F}$ Mohamed. ${ }^{1}$ Faculty of Medicine, University of Southampton, Southampton, UK; ${ }^{2}$ Pseudomyxoma Centre, Basingstoke and North Hampshire NHS Foundation Trust, Basingstoke, UK; ${ }^{3}$ Histopathology, Basingstoke and North Hampshire NHS Foundation Trust, Basingstoke, UK; ${ }^{4}$ Department of Surgery, University Hospitals Southampton, Southampton, UK

Introduction The current WHO classification of tumours of the digestive system divides pseudomyxoma peritonei into two grades, namely low-grade and high-grade. This study was designed to correlate survival with low-grade and high-grade pseudomyxoma peritonei classified according to the WHO criteria.

Methods The histological slides of 274 consecutive patients were reviewed and designated as either low-grade or high-grade. The patients had been referred for cytoreductive surgery. The grade of the pseudomyxoma was correlated with survival data using the Kaplan-Meier method with the log-rank (Mantel-Cox) test.

Results 238 (87\%) patients had low-grade lesions and 36 (13\%) had high-grade lesions. The most common primary tumour was a low grade appendiceal mucinous neoplasm (231 cases, 84\%). Seven patients who died within 30 days of their operation (a postoperative mortality of $2.6 \%$ ) were excluded from survival analysis. Another patient was excluded because of incomplete survival data. The remaining 266 patients showed an overall 5-year survival of $63 \%$ in patients with low-grade pseudomyxoma peritonei and $23 \%$ in patients with high-grade pseudomyxoma peritonei $(p<0.001)$. Complete cytoreduction was achieved in 165 (60\%) patients; the 5year survival for low-grade and high-grade was $84 \%$ and $48 \%$ respectively in this group $(p<0.001)$. The median survival of patients who had complete cytoreduction was 7.7 years for lowgrade and 2.8 years for high-grade $(\mathrm{p}<0.001)$.

Conclusion Histological classification of pseudomyxoma peritonei as low-grade or high-grade correlates with prognosis. This may identify a group who could benefit from further adjuvant therapy which is not generally advocated for appendiceal mucinous tumours.

Competing interests None declared.

\section{PWE-098 CLOSTRIDIUM DIFFICILE DIARRHOEA-THE CHANGING HOSPITAL EPIDEMIOLOGY AND CLINICAL OUTCOMES FROM A HIGH PREVALENCE AREA IN NORTH EAST ENGLAND}

doi:10.1136/gutjnl-2012-302514d.98

${ }^{1} \mathrm{~J}$ Ross, ${ }^{1} \mathrm{P}$ Brown, ${ }^{*} \mathrm{C}$ Aldridge, ${ }^{2} \mathrm{~L}$ Lim, ${ }^{2} \mathrm{D}$ Nayar, ${ }^{3} \mathrm{~J}$ Sloss, ${ }^{2} \mathrm{D}$ Allison, ${ }^{1} \mathrm{~A}$ Dhar. ${ }^{1}$ Department of Gastroenterology, County Durham \& Darlington NHS Foundation Trust, Bishop Auckland, UK; ${ }^{2}$ Department of Microbiology, County Durham \& Darlington NHS Foundation Trust, Durham, UK; ${ }^{3}$ Department of Microbiology, County Durham \& Darlington NHS Foundation Trust, Darlington, UK

Introduction Clostridium difficile associated diarrhoea (CDAD) is an important hospital acquired infection. In 2008-2009 Co. Durham had one of the highest reported annual incidence of CDAD with 232 cases, 74.4 cases $/ 100000$ bed days. Following strict antibiotic stewardship in 2009, we set out to examine the changes to the hospital based epidemiology of CDAD in our three hospitals over a 12-month period.

Methods Between June 2010 and May 2011, 70 patients with positive stool $C$ difficile toxin were identified from the Microbiology database, and 56 case notes reviewed. Patient demographics, clinical symptoms, risk factors, severity, treatment for CDAD, and multidisciplinary team decisions were recorded. Clinical outcomes 